\title{
Combining Ability Analysis for Yield and Yield Contributing Traits in Maize (Zea mays L.) Hybrids
}

\author{
G. B. Bharat ${ }^{1}$, P. B. Wadikar ${ }^{1}$, M. V. Dhuppe ${ }^{2}$ and N. R. Thakur $^{3}$ \\ ${ }^{1}$ Department of Agricultural Botany, College of Agriculture, Latur, India \\ ${ }^{2}$ Oilseed Research Station, Latur, India \\ ${ }^{3}$ Department of Agricultural Botany, College of Agriculture, Parbhani, India \\ *Corresponding author
}

\begin{abstract}
A B S T R A C T

\begin{tabular}{|l|}
\hline Ke y w or d s \\
Maize, Combining \\
ability, GCA and \\
SCA
\end{tabular}

Study on combining ability effects were carried out in 28 hybrids obtained by crossing $8 \times 8$ genotypes in half diallel fashion and parents, hybrids evaluated with standard check Maharaja in RBD with three replication during kharif2018-19 at research farm of Agriculture botany College of Agriculture, Latur for twelve traits viz., days to 50 per cent tasseling, days to 50 per cent silking, days to maturity, plant height $(\mathrm{cm})$, ear length $(\mathrm{cm})$, ear girth $(\mathrm{cm})$, kernel row number, number of grains per row, 100-grain weight $(\mathrm{g})$, fodder yield per plant $(\mathrm{g})$, grain yield per plant $(\mathrm{g})$ and Harvest index $(\%)$. The analysis of variance revealed that significant differences among treatments, parents, hybrids and parents $v / s$ hybrids for all characters except days to 50 per cent tasseling and days to 50 percent silking. This validated that, considerable amount of variability among experimental material. The combining ability analysis revealed the presence of higher magnitude of SCA than GCA variance for all characters under study. The ratio of additive to dominance variance was lower than unity for all traits, indicating higher non-additive variance than additive variance. Among parents, 231-A, Hyd.19051 and Hyd.19051 was found to good general combiner for grain yield per plant. While the parent 231A exhibited good general combining ability for majority of characters. Best specific combinations were Hyd.18227 x Hyd.08R-740-2, Hyd.19051 x Hyd.12288, Hyd.18227 x Hyd.18169 and Hyd.18063 x Hyd.18169 for grain yield per plant.
\end{abstract}

\section{Introduction}

Maize is third most important cereal crop after rice and wheat in the world. It is referred as the "Queen of Cereals" and "Miracle Crop" due to its high productivity potential as compared to the other cereals. Being a potential field crop in India, maize occupies an important place as a source of human food (26\%), animal feed (11\%), poultry feed $(43 \%)$, industrial products $(12 \%)$ and three per cent for seed and other purposes (FICCI). Many forms of maize are used i.e. Flour corn, Popcorn, Flint corn, Dent corn, Waxy corn, Sweet corn, Pod corn and Stripped maize.

India is the fifth largest producer of maize in the world contributing $3 \%$ of the total global production. It is cultivated on nearly 150 million ha in about 160 countries having wider diversity of soil, climate, biodiversity and management practices that contributes 
$36 \%$ (782 MT) of the global grain production. The United States of America (USA) is the largest producer of maize contributes nearly $35 \%$ of the total production in the world. The important maize growing countries are USA, China, Brazil, Mexico, India, Philippines, South Africa and Indonesia.

In India, major maize growing states are Karnataka, Madhya Pradesh, Maharashtra, Rajasthan, Bihar, Uttar Pradesh, Telangana, Gujarat and Tamil Nadu. In India, maize has been grown in an area of 9.2 million ha with production of 24.17 MT and average productivity of $2.56 \mathrm{t} / \mathrm{ha}$.

Being a highly cross pollinated crop, it offers tremendous scope for the plant breeders for genetic improvement. It possesses one of the most well studied genetic systems among cereals which have motivated a rich history of research into the genetics of various traits in maize. Information on different traits of interest especially their genetic control is prerequisite for planning the genetic improvement strategies.

The main goal of maize breeding is to obtain new hybrids with high genetic potential for yield and positive features that exceed the existing commercial hybrids. The commercial production of hybrids however, depends upon two factors viz., the behavior of the line itself and the behavior of line in hybrid combination. The behavior of a line in hybrid combination is assessed through the estimation of combining ability effects.

Sprague and Tatum (1942) coined combining ability to designate the average performance of line in hybrid combination and to define those cases in which certain combination do relatively better or worse than would be expected on the basis of the average performance of the lines involved. Diallel crossing program have been applied to achieve this goal by providing a systematic approach for detection of suitable parents and crosses for the investigated characters.

\section{Materials and Methods}

Total eight maize inbred lines (10306, Hyd.19051, Hyd.12288, Hyd.18227, Hyd.18063, Hyd.18169, Hyd.08R-740-2, 231A) were used as parents in $8 \times 8$ half diallel crossing scheme. All the possible crosses $n(n-$ 1)/2 excluding reciprocals were effected in a half diallel mating design to produce 28 hybrids during summer 2018 at Experimental Farm, Department of Agril. Botany, College of Agriculture, Latur. Seeds were sown in the field by keeping plant to plant and row to row distances $20 \mathrm{~cm}$ and $60 \mathrm{~cm}$ respectively.

At maturity all the parents were crossed following half diallel mating design, resultantly 28 F1s were generated. Seeds harvested from F1s, parents and standard check (Maharaja) were sown by following randomized block design (RBD) with three replications. All the agronomic practices were taken for establishment of proper crop stand in field.

Data were recorded for yield components i.e. days to 50 per cent tasseling, days to 50 per cent silking, days to maturity, plant height $(\mathrm{cm})$, ear length $(\mathrm{cm})$, ear girth $(\mathrm{cm})$, kernel row number, number of grains per row, 100grain weight $(\mathrm{g})$, fodder yield per plant $(\mathrm{g})$, grain yield per plant $(\mathrm{g})$ and harvest index $(\%)$.The observations were recorded on randomly selected five plants and subjected to statistical analysis. Analysis of variance technique suggested by Panse and Sukhatme (1967) was followed to test the differences among the genotypes for all the characters. Combining ability analysis was performed with the data obtained for parents and hybrids according to Model-I, Method-II proposed by Griffing (1956). 


\section{Results and Discussion}

\section{Analysis of variance}

The analysis of variance for randomized block design was carried out (Table. 1) for the character of yield and its components to see the ignorance of differences among the treatments, replications and other partitioning source of variation. The analysis of variance revealed that, the all characters i.e. days to $50 \%$ tasseling, days to $50 \%$ silking, days to maturity, plant height $(\mathrm{cm})$, ear length $(\mathrm{cm})$, ear girth $(\mathrm{cm})$, kernel row number, no. of grains per row, 100 grain weight, fodder yield per plant (g), grain yield per plant (g), harvesting index $(\%)$ had showed significant differences between treatments as well as parents. Parents $v / s \quad F_{1} s$, were significant all the characters except days to $50 \%$ tasseling and days to $50 \%$ silking. Similar results reported by Jawahar and Kumar(2012) and Ratna et al., (2014).

\section{Combining ability effects}

The knowledge on combining ability assists in the selection of suitable parental line. Among various biometrical techniques available, combining ability analysis proposed by Griffing (1956) had been extensively used by breeders. It provides information on the performance of genotypes in hybrid combination and also nature of gene action involved in the control of metric traits.

\section{General combining ability effects}

The general combining ability effects of eight parents for twelve traits were presented in Table 3. Among the eight parents studied, Hyd.12288 (-1.621), Hyd.10306 (-0.654) and Hyd.19051 (-0.454) expressed significant and desirable negative GCA effects and proved to be good general combiner for days to tassseling while parents, Hyd.12288 (-1.258),
Hyd.08R-740-2 (-0.942), Hyd.10306 (-0.792) and Hyd.19051 (-0.492) expressed significant and desirable negative GCA effects and proved to be good general combiner for days to silking. For days to maturity the parents Hyd.10306 (-1.207), Hyd.18063 (-1.117) and Hyd.12288 (-0.150) shows negatively significant GCA effects.

The negative significant GCA effects was registered for ear height by three parents Hyd.10306 (-5.71), 231-A (-3.58) and Hyd.18169 (-2.11). While two parents viz., Hyd.10306 (0.352) and 231-A (0.795) depicted significant and positive GCA effects for ear length. For ear girth parent Hyd.19051 (0.421) exhibited highest positively significant GCA effect.

The trait kernel row number was recorded significant positive GCA effects in two parents 231-A (0.439) and Hyd.12288 (0.366) while parent, 231-A (1.545) explored highest positive significant GCA effect for number of grains per row. Among the parents, the parent Hyd.19051 (2.30) exhibited highest positively significant GCA effect for 100 grain weight. For fodder yield per plant parents, Hyd.18169 (5.792) and Hyd.18063 (2.225) were depicted positive significant GCA effects. The parent 231-A manifested highest, significant and positive GCA effects (7.408) followed by Hyd.19051 (6.342) and Hyd.19051 (4.842) respectively for grain yield per plant. Among the eight parents, three parents showed positively significant GCA effects for harvesting index. Out of eight parents, 231-A was found to good general combiner for eight characters including grain yield per plant and the parents, Hyd.19051 and Hyd.12288 for seven characters. The high positive value of GCA effect of these parents indicates that their contribution in transferring those traits to their hybrids is high. This is in accordance with findings of Gosai et al., (2017), Begum et al., (2018) and Karim et al., (2018). 
Table.1 Analysis of variance for different characters in maize

\begin{tabular}{|c|c|c|c|c|c|c|c|c|c|c|c|c|c|}
\hline Source & D.F. & $\begin{array}{c}\text { Days to } \\
50 \% \\
\text { tasseling }\end{array}$ & $\begin{array}{c}\text { Days to } \\
50 \% \\
\text { silking }\end{array}$ & $\begin{array}{c}\text { Days to } \\
\text { maturity }\end{array}$ & $\begin{array}{c}\text { Plant } \\
\text { height } \\
\text { (cm) }\end{array}$ & $\begin{array}{c}\text { Ear } \\
\text { length } \\
(\mathrm{cm})\end{array}$ & $\begin{array}{l}\text { Ear } \\
\text { girth } \\
(\mathrm{cm})\end{array}$ & $\begin{array}{l}\text { Kernel } \\
\text { row } \\
\text { number }\end{array}$ & $\begin{array}{c}\text { No. of } \\
\text { grains } \\
\text { per row }\end{array}$ & $\begin{array}{c}100 \\
\text { grain } \\
\text { weight } \\
\text { (g) }\end{array}$ & $\begin{array}{c}\text { Fodder } \\
\text { yield per } \\
\text { plant }(g)\end{array}$ & $\begin{array}{c}\text { Grain yield } \\
\text { per plant } \\
(\mathrm{g})\end{array}$ & $\begin{array}{c}\text { Harvesting } \\
\text { index }(\%)\end{array}$ \\
\hline Replications & 2 & 0.210 & 0.009 & 1.009 & 6.953 & 0.035 & 0.015 & 1.313 & 0.826 & 0.720 & 0.481 & 11.861 & 0.335 \\
\hline Treatments & 35 & $14.575^{* *}$ & $13.831 * *$ & $38.716^{* *}$ & $519.433 * *$ & $9.547 * *$ & $2.853 * *$ & $3.076^{* *}$ & $44.066^{* *}$ & $39.461 * *$ & $256.879 * *$ & $1251.188 * *$ & $77.029 * *$ \\
\hline Parents & 7 & $16.946^{* *}$ & $18.755^{* *}$ & $34.089 * *$ & $706.755 * *$ & $5.997 * *$ & $1.943 * *$ & $2.272 * *$ & $18.372 * *$ & $9.787 * *$ & $103.184 * *$ & $518.547 * *$ & $48.377 * *$ \\
\hline $\mathrm{F}_{1}{ }^{\prime} \mathrm{s}$ & 27 & $14.465^{* *}$ & $13.067 * *$ & $40.791 * *$ & $458.55^{* *}$ & $9.524 * *$ & $2.396 * *$ & $2.277 * *$ & $46.348 * *$ & $48.525^{* *}$ & $299.070 * *$ & $1321.373^{* *}$ & $76.458 * *$ \\
\hline $\begin{array}{c}\text { Parents v/s } \\
F_{1}^{\prime} s\end{array}$ & 1 & 0.955 & 0.001 & $15.080 * *$ & $852.000 * *$ & $35.017 * *$ & $21.559 * *$ & $30.260 * *$ & $162.315^{* *}$ & $69.711 * *$ & $193.572 * *$ & $4484 . .666 * *$ & $293.004 * *$ \\
\hline Error & 70 & 1.348 & 1.409 & 1.685 & 30.134 & 0.628 & 0.093 & 0.530 & 1.118 & 1.009 & 17.34 & 53.880 & 2.85 \\
\hline
\end{tabular}

* Significant at $5 \%$ level, ** Significant at $1 \%$ level

Table.2 Analysis of variance of combining ability for different characters in maize

\begin{tabular}{|c|c|c|c|c|c|c|c|c|c|c|c|c|c|}
\hline Source & $\begin{array}{l}\text { D. } \\
\text { F. }\end{array}$ & $\begin{array}{c}\text { Days to } \\
50 \% \\
\text { tasseling }\end{array}$ & $\begin{array}{c}\text { Days to } \\
50 \% \\
\text { silking }\end{array}$ & $\begin{array}{l}\text { Days to } \\
\text { maturity }\end{array}$ & $\begin{array}{c}\text { Plant } \\
\text { height } \\
\text { (cm) }\end{array}$ & $\begin{array}{c}\text { Ear } \\
\text { length } \\
\text { (cm) }\end{array}$ & $\begin{array}{l}\text { Ear girth } \\
\quad(\mathbf{c m})\end{array}$ & $\begin{array}{c}\text { Kernel } \\
\text { row } \\
\text { number }\end{array}$ & $\begin{array}{c}\text { No. of } \\
\text { grains } \\
\text { per row }\end{array}$ & $\begin{array}{l}100 \text { grain } \\
\text { weight (g) }\end{array}$ & $\begin{array}{l}\text { Fodder } \\
\text { yield per } \\
\text { plant (g) }\end{array}$ & $\begin{array}{l}\text { Grain yield } \\
\text { per plant (g) }\end{array}$ & $\begin{array}{c}\text { Harvestin } \\
\text { g index } \\
(\%)\end{array}$ \\
\hline GCA & 7 & 7.704** & $8.802 * *$ & $7.994 * *$ & $191.302 * *$ & $2.496 * *$ & $1.187 * *$ & $0.781 * *$ & $13.798 * *$ & $26.955 * *$ & $79.872 * *$ & $389.485^{* *}$ & $26.388 * *$ \\
\hline SCA & 28 & $4.147 * *$ & $3.563 * *$ & $14.133 * *$ & $168.605^{* * *}$ & $3.354 * *$ & $0.892 * *$ & $1.086^{* *}$ & $14.912 * *$ & $9.704 * *$ & $87.065^{* *}$ & $423.957 * *$ & $25.499 * *$ \\
\hline Error & 70 & 0.450 & 0.470 & 0.562 & 10.045 & 0.209 & 0.031 & 0.177 & 0.373 & 0.336 & 5.783 & 17.960 & 0.951 \\
\hline $\begin{array}{l}\delta 2 \\
\text { gca/sca }\end{array}$ & & 0.096 & 0.169 & 0.045 & 0.014 & 0.027 & 0.034 & 0.033 & 0.007 & 0.184 & 0.008 & 0.008 & 0.003 \\
\hline
\end{tabular}


Table.3 The general combining ability effects of eight parents for twelve traits

\begin{tabular}{|c|c|c|c|c|c|c|c|c|c|c|c|c|c|}
\hline $\begin{array}{l}\text { Sr. } \\
\text { No. }\end{array}$ & Parents/ crosses & $\begin{array}{c}\text { Days to } \\
50 \% \\
\text { tasseling }\end{array}$ & $\begin{array}{c}\text { Days to } \\
50 \% \\
\text { silking }\end{array}$ & $\begin{array}{l}\text { Days to } \\
\text { maturity }\end{array}$ & $\begin{array}{l}\text { Plant height } \\
(\mathrm{cm})\end{array}$ & $\begin{array}{l}\text { Ear length } \\
\quad(\mathrm{cm})\end{array}$ & $\begin{array}{l}\text { Ear } \\
\text { girth } \\
(\mathrm{cm})\end{array}$ & $\begin{array}{c}\text { Kernel } \\
\text { row } \\
\text { number }\end{array}$ & $\begin{array}{c}\text { No. of } \\
\text { grains } \\
\text { per row }\end{array}$ & $\begin{array}{l}100 \text { grain } \\
\text { weight }(g)\end{array}$ & $\begin{array}{l}\text { Fodder } \\
\text { yield per } \\
\text { plant (g) }\end{array}$ & $\begin{array}{l}\text { Grain } \\
\text { yield per } \\
\text { plant (g) }\end{array}$ & $\begin{array}{c}\text { Harvestin } \\
\text { g index } \\
(\%)\end{array}$ \\
\hline & Parents & & & & & & & & & & & & \\
\hline 1 & Hyd.10306 & $-0.654 * *$ & $-0.792 * *$ & $-1.207 * *$ & $-5.717 * *$ & $0.352 *$ & $-0.142 * *$ & -0.218 & $1.525 * *$ & $-1.186^{* *}$ & $-1.775^{*}$ & -0.558 & 0.253 \\
\hline 2 & Hyd.19051 & $-0.454 * *$ & $-0.492 *$ & -0.017 & $3.983^{* *}$ & 0.028 & $0.421 * *$ & -0.024 & -0.275 & $2.301 * *$ & $-2.875^{* *}$ & $6.342 * *$ & $1.903 * *$ \\
\hline 3 & Hyd.12288 & $-1.621 * *$ & $-1.258^{* *}$ & -0.150 & -1.550 & -0.095 & $0.408 * *$ & $0.366 * *$ & $0.568 * *$ & -0.236 & 0.592 & $4.842 * *$ & $1.034 * *$ \\
\hline 4 & Hyd.18227 & $0.412 * *$ & -0.058 & $0.983^{* *}$ & $6.883^{* *}$ & $-0.925^{* *}$ & -0.009 & $-0.247 *$ & 0.072 & 0.041 & -0.808 & 1.108 & 0.134 \\
\hline 5 & Hyd. 18063 & $0.863 * *$ & $1.675^{* *}$ & $-1.117 * *$ & $3.883^{* *}$ & 0.048 & $-0.195 * *$ & -0.157 & $-0.888 * *$ & $-2.266^{* *}$ & $2.225^{* * *}$ & $-10.258^{* *}$ & $-2.553 * *$ \\
\hline 6 & Hyd. 18169 & 0.062 & -0.092 & -0.083 & $-2.117 * *$ & $-0.328 *$ & $-0.112^{*}$ & 0.112 & $-1.778 * *$ & $0.421 *$ & $5.792 * *$ & -2.458 & $-1.672 * *$ \\
\hline 7 & Hyd.08R-740-2 & $1.046 * *$ & $-0.942 * *$ & 0.050 & -1.783 & 0.125 & $-0.602 * *$ & $-0.271^{*}$ & $-0.768 * *$ & $-1.323 * *$ & $-1.442 *$ & $-6.425 * *$ & $-0.960 * *$ \\
\hline \multirow[t]{3}{*}{8} & 231-A & 0.346 & 0.075 & $1.450 * *$ & $-3.583^{* *}$ & $0.795 * *$ & $0.231 * *$ & $0.439 * *$ & $1.545^{* *}$ & $2.248^{* *}$ & $-1.708^{*}$ & $7.408 * *$ & $1.862 * *$ \\
\hline & S.E.gi & 0.395 & 0.404 & 0.442 & 1.869 & 0.270 & 0.104 & 0.248 & 0.360 & 0.342 & 1.418 & 2.500 & 0.575 \\
\hline & S.E.gi-gj & 0.598 & 0.611 & 0.668 & 2.826 & 0.408 & 0.157 & 0.375 & 0.544 & 0.517 & 2.144 & 3.779 & 0.869 \\
\hline \multicolumn{2}{|c|}{ Crosses } & & & & & & & & & & & & \\
\hline 9 & $\begin{array}{l}\text { Hyd.10306 x } \\
\text { Hyd.19051 }\end{array}$ & $3.159 * *$ & $2.070 * *$ & -0.441 & $11.863 * *$ & $2.759 * *$ & $1.170 * *$ & 0.489 & $3.081 * *$ & -0.210 & 0.863 & $14.744 * *$ & 2.652 \\
\hline 10 & $\begin{array}{l}\text { Hyd.10306 x } \\
\text { Hyd.12288 }\end{array}$ & 0.326 & -0.163 & $3.026 * *$ & 5.396 & $1.349 * *$ & $0.684 * *$ & 0.632 & $2.438 * *$ & $-2.674 * *$ & $-12.270 * *$ & 3.911 & $3.252 *$ \\
\hline 11 & $\begin{array}{l}\text { Hyd.10306 x } \\
\text { Hyd.18227 }\end{array}$ & $-3.374 * *$ & $-3.363 * *$ & $-4.441 * *$ & $10.296 * *$ & $-1.448 * *$ & $-1.066^{* *}$ & $-1.321 * *$ & $-4.065 * *$ & $-4.217 * *$ & $10.463^{* *}$ & $-39.689 * *$ & $-11.166^{* *}$ \\
\hline 12 & $\begin{array}{l}\text { Hyd.10306 x } \\
\text { Hyd.18063 }\end{array}$ & -0.157 & 0.237 & $-4.341 * *$ & $-16.704 * *$ & 0.506 & 0.187 & 0.189 & $2.628 * *$ & $-2.477 * *$ & $11.430 * *$ & -5.322 & $-2.325 * *$ \\
\hline 13 & $\begin{array}{l}\text { Hyd.10306 x } \\
\text { Hyd.18169 }\end{array}$ & $-3.357 * *$ & $-1.663 *$ & $1.959 * *$ & 1.963 & 0.049 & -0.230 & 0.119 & -0.815 & $-1.364^{*}$ & -1.137 & 2.211 & 0.871 \\
\hline 14 & $\begin{array}{l}\text { Hyd.10306 x Hyd.08R- } \\
740-2\end{array}$ & $2.326 * *$ & $1.304 *$ & $5.826^{* *}$ & $-6.370 *$ & -0.504 & $0.660 * *$ & 0.402 & $-4.959 * *$ & $1.746 * *$ & $13.763 * *$ & $-8.156^{*}$ & $-3.025 * *$ \\
\hline 15 & Hyd.10306 x 231-A & -0.307 & -0.496 & 1.093 & 4.430 & 0.459 & $1.260^{* *}$ & 0.526 & $5.128 * *$ & 0.510 & $-15.304 * *$ & $11.678^{* *}$ & $3.817 *$ \\
\hline 16 & $\begin{array}{l}\text { Hyd.19051 x } \\
\text { Hyd.12288 }\end{array}$ & $-1.874 * *$ & -0.796 & $-4.074 * *$ & -4.304 & $2.072 * *$ & 0.120 & -0.528 & $4.738 * *$ & $2.840 * *$ & $10.496^{* *}$ & $29.678^{* *}$ & $4.025^{* *}$ \\
\hline 17 & $\begin{array}{l}\text { Hyd.19051 x } \\
\text { Hyd.18227 }\end{array}$ & $-1.907 * *$ & $-1.663^{*}$ & $4.126^{* *}$ & $-17.404 * *$ & 0.002 & -0.130 & $-0.814^{*}$ & $-3.432 * *$ & $2.563^{* *}$ & -3.104 & -6.589 & -0.353 \\
\hline 18 & Hyd.19051 x & -0.024 & -0.396 & $-4.441 * *$ & 5.263 & -0.304 & $1.190 * *$ & $1.029 *$ & $1.661 * *$ & $1.370^{*}$ & 0.863 & $14.444 * *$ & 2.851 \\
\hline
\end{tabular}




\section{Int.J.Curr.Microbiol.App.Sci (2020) 9(6): 1234-1241}

\begin{tabular}{|c|c|c|c|c|c|c|c|c|c|c|c|c|c|}
\hline & Hyd.18063 & & & & & & & & & & & & \\
\hline 19 & $\begin{array}{l}\text { Hyd.19051 x } \\
\text { Hyd. } 18169\end{array}$ & 0.443 & 0.704 & -0.807 & 4.596 & 0.206 & 0.140 & -0.408 & -1.049 & $5.383 * *$ & -1.370 & $13.644 * *$ & $3.007 *$ \\
\hline 20 & $\begin{array}{l}\text { Hyd.19051 x Hyd.08R- } \\
740-2\end{array}$ & -0.541 & $-1.330^{*}$ & $4.393^{* *}$ & -1.737 & $-2.981 * *$ & $-0.470^{*}$ & $1.042 *$ & $-1.692 * *$ & $-2.840 * *$ & -4.470 & $-14.722 * *$ & $-2.282 * *$ \\
\hline 21 & Hyd.19051 x 231-A & $-1.841^{* *}$ & -0.130 & $2.326^{* *}$ & -3.270 & $1.816^{* *}$ & $0.297 *$ & -0.268 & $3.261 * *$ & -0.110 & $-12.537 * *$ & -0.222 & 2.563 \\
\hline 22 & $\begin{array}{l}\text { Hyd.12288 x } \\
\text { Hyd.18227 }\end{array}$ & 0.593 & 0.104 & 0.926 & -5.204 & $-1.174 *$ & $-1.216^{* *}$ & -0.404 & -0.342 & $1.533 * *$ & 1.430 & -3.089 & -0.700 \\
\hline 23 & $\begin{array}{l}\text { Hyd. } 12288 \text { x } \\
\text { Hyd. } 18063\end{array}$ & $4.809^{* *}$ & $5.370 * *$ & $5.026^{* *}$ & $-17.537 * *$ & $-1.348 * *$ & 0.304 & -0.194 & 0.618 & $-2.707 * *$ & -3.604 & $-9.389 *$ & -1.443 \\
\hline 24 & $\begin{array}{l}\text { Hyd.12288 x Hyd. } \\
18169\end{array}$ & $1.276^{*}$ & $2.470^{* *}$ & $1.659^{*}$ & -5.537 & $0.762^{*}$ & $0.687^{* *}$ & $1.269^{* *}$ & $-1.592^{* *}$ & 0.753 & $10.830^{* *}$ & -1.856 & -1.500 \\
\hline 25 & $\begin{array}{l}\text { Hyd. } 12288 \text { x Hyd.08R- } \\
740-2\end{array}$ & $1.293^{*}$ & 0.104 & 0.526 & $11.796^{* *}$ & -0.858 & 0.110 & -0.181 & 0.731 & $-1.570 * *$ & $-12.604 * *$ & $-6.889 * *$ & 1.034 \\
\hline 26 & Hyd.12288 x 231-A & $-3.007 * *$ & $-1.696^{*}$ & $2.126^{* *}$ & $-6.404 *$ & $1.339 * *$ & $0.310^{*}$ & $0.909 *$ & 0.618 & $2.460 * *$ & 4.330 & $15.278^{* *}$ & 1.706 \\
\hline 27 & $\begin{array}{l}\text { Hyd.18227 x } \\
\text { Hyd.18063 }\end{array}$ & -0.891 & $-1.496^{*}$ & $-2.441 * *$ & 2.363 & $-1.551 * *$ & -0.080 & 0.586 & $-2.785 * *$ & $-2.037 * *$ & $5.130 *$ & -7.656 & $-2.387 * *$ \\
\hline 28 & $\begin{array}{l}\text { Hyd. } 18227 \text { x } \\
\text { Hyd. } 18169\end{array}$ & $1.909 * *$ & 0.604 & $-3.474 * *$ & $11.363 * *$ & $1.426^{* *}$ & $1.304 * *$ & $0.982 *$ & $6.238 * *$ & -0.690 & -3.770 & $26.878 * *$ & $6.069^{* *}$ \\
\hline 29 & $\begin{array}{l}\text { Hyd. } 18227 \text { x Hyd.08R- } \\
740-2\end{array}$ & $-1.407 *$ & -0.096 & $-1.941 * *$ & 0.030 & $5.572^{* *}$ & $2.027 * *$ & $2.299^{* *}$ & $9.628 * *$ & $2.920^{* *}$ & $-15.537 * *$ & $54.178 * *$ & $12.990 * *$ \\
\hline 30 & Hyd.18227 x 231-A & 0.626 & 0.104 & 0.993 & $-24.170 * *$ & $-1.098^{*}$ & $-2.240 * *$ & -0.478 & $-4.019 * *$ & $2.283^{* *}$ & 2.396 & -0.656 & -0.218 \\
\hline 31 & $\begin{array}{l}\text { Hyd. } 18063 \mathrm{x} \\
\text { Hyd. } 18169\end{array}$ & $-1.707 *$ & $-1.463^{*}$ & $-1.707^{*}$ & $13.696^{* *}$ & $1.652 * *$ & 0.157 & 0.226 & $1.531 * *$ & $3.583 * *$ & $-7.804 *$ & $19.578^{* *}$ & $5.723^{* *}$ \\
\hline 32 & $\begin{array}{l}\text { Hyd. } 18063 \text { x Hyd.08R- } \\
740-2\end{array}$ & 0.476 & $1.837 * *$ & $-6.841 * *$ & -0.970 & $-1.268 * *$ & $0.780 * *$ & $1.276^{* * *}$ & -0.745 & $-2.207 * *$ & $-16.904 * *$ & -2.789 & 2.591 \\
\hline 33 & Hyd. 18063 x 231-A & -1.157 & $-1.630^{*}$ & $3.426^{* *}$ & $11.830 * *$ & 0.429 & $-0.853 * *$ & 0.232 & $2.608 * *$ & $1.590 * *$ & 1.363 & $11.711 * *$ & 2.256 \\
\hline 34 & $\begin{array}{l}\text { Hyd.18169 x Hyd.08R- } \\
740-2\end{array}$ & -0.057 & -0.396 & $3.459^{* * *}$ & 5.696 & 0.209 & $-1.136^{* *}$ & -0.694 & -0.589 & $-4.894 * *$ & $7.863 *$ & $-28.589 * *$ & $-7.920 * *$ \\
\hline 35 & Hyd.18169 x 231-A & $1.643^{*}$ & $1.804 * *$ & $1.726^{*}$ & -3.837 & 0.506 & $0.997 * *$ & $1.396^{* *}$ & 1.098 & $3.036^{* *}$ & -3.302 & $17.911 * *$ & $4.502 * *$ \\
\hline \multirow[t]{3}{*}{36} & $\begin{array}{l}\text { Hyd.08R-740-2 x 231- } \\
\text { A }\end{array}$ & $1.326^{*}$ & 0.104 & $2.074 * *$ & $-29.170 * *$ & -0.014 & -0.280 & -0.388 & $-3.579 * *$ & $-2.254 * *$ & $12.363 * *$ & -3.789 & -1.934 \\
\hline & S.E sij & 1.212 & 1.239 & 1.355 & 5.731 & 0.827 & 0.319 & 0.760 & 1.104 & 1.048 & 4.348 & 7.664 & 1.763 \\
\hline & S.E sij - sik & 1.794 & 1.833 & 2.005 & 8.48 & 1.224 & 0.472 & 1.125 & 1.663 & 1.551 & 6.434 & 11.339 & 2.609 \\
\hline
\end{tabular}




\section{Specific combining ability effects}

The specific combining ability effects of 28 direct crosses with respect to twelve traits presented in Table 3. The estimates of the SCA effects of 28 hybrids ranged from 39.689 (Hyd.10306 x Hyd.18227) to 54.178 (Hyd.18227 x Hyd.08R-740-2) for grain yield per plant. The cross, Hyd.10306 x Hyd.18227 (-3.374) expressed highest significant and negative SCA effect for days to tasseling and days to silking. For days to maturity highest significant negative SCA recorded by the cross, Hyd.18063 x Hyd.08R-740-2 (-6.841) followed by Hyd.10306 x Hyd.18227 (-4.441) and Hyd.19051 x Hyd.18063 (-4.441).

Highest significant positive SCA recorded by the cross, Hyd.18063 x Hyd.18169 (13.696) for plant height followed by Hyd.10306 x Hyd.19051 (11.863) and Hyd.18063 x 231-A (11.830). For ear length and ear girth highest SCA effect recorded by the cross, Hyd.18227 $x$ Hyd.08R-740-2 (5.572). For kernel row number out of 28 crosses, eight showed positively significant SCA effect.

The cross, Hyd.18227 x Hyd.08R-740-2 displayed maximum SCA effect (2.299) followed by Hyd.18169 x 231-A (1.396) and Hyd.18063 x Hyd.08R-740-2 (1.276). For number of grains per row Hyd.18227 x Hyd.08R-740-2 (9.628) had exhibited highest positively significant SCA effect. Among the hybrids, the cross Hyd.19051 x Hyd.18169 (5.383) was showed highest positively significant SCA effects for 100 grain weight followed by Hyd.18063 x Hyd.18169 (3.583) and Hyd.18169 x 231-A (3.036) respectively. For fodder yield per plant out of 28 crosses, eight showed positively significant SCA effect. The cross, Hyd.10306 x Hyd.08R-7402 (13.763) displayed maximum SCA effect. For grain yield per plant, SCA effect fluctuated between -39.689 (Hyd.10306 x Hyd.18227) to 54.178 (Hyd.18227 x
Hyd.08R-740-2).

Among the 28 crosses, eleven crosses depicted significant and positive SCA effect under study. The cross, Hyd.18227 $\mathrm{x}$ Hyd.08R-740-2 (54.178) registered highest significant positive SCA followed by Hyd.19051 x Hyd.12288 (29.678), Hyd.18227 x Hyd.18169 (26.878) and Hyd.18063 x Hyd.18169 (19.578) respectively. For harvesting index Hyd.18227 x Hyd.08R-7402 (12.990) had exhibited highest positively significant SCA effect followed by Hyd.18227 x Hyd.18169 (6.069) and Hyd.18063 x Hyd.18169 (5.723).

Good general combining inbred parents have not always showed high SCA effects in their cross combination. Thus it may be concluded that the information on GCA effects alone may not be sufficient to predict the extent of hybrid vigour by a particular cross combination. In many crosses significant SCA effects for yield per plant were associated with negative SCA effects of days to maturity.

This is desirable because we want hybrids having high yield and early in crop duration (Yerva et al., 2016). In general, crosses involving both good general combiners as well as one good and other poor combiner showed high SCA effects, which is due to additive $\mathrm{x}$ additive and dominance $\mathrm{x}$ dominance gene action. Evaluation of parental inbreds together with per se and GCA effects resulted in the identification of 231-A as the best parent. The estimates of SCA effects of hybrids revealed that the hybrids Hyd.18227 x Hyd.08R-740-2 (54.178), Hyd.19051 x Hyd.12288 (29.678), Hyd.18227 x Hyd.18169 (26.878) and Hyd.18063 x Hyd.18169 (19.578) registered significant and favorable SCA effects for majority of traits including grain yield per plant. 


\section{References}

Begum, S. S., Alam, S. H., Omy, M. A. and Rohman, M. M. (2018). Inheritance and combing ability in maize using a $7 \times 7$ diallel cross. J. of Plant Breeding and Crop Sci., 10(9): 239-248.

Griffings, B. (1956). Concept of general and specific combining ability relation to diallel crossing system. Aust. J. Bil. Sci., 9:463-493.

Gosai, M. A., Kuchhadiya, G. V., Brahmbhatt, B. N. and Bhalala, K. N. (2017). Study of combining ability in diallel crosses of maize (Zea mays L.) for grain yield and quality traits. Int. J. Res. Agric. Sci., 4(2): 2348-3997.

Jawahar, J. L. and Kumar, R. S. (2012). Combining ability and heterosis for polygenic characters in maize (Zea mays L.). Madras Agric. J., 99(4-6): 174-177.

Karim, A. N. M. S., Ahmed, S., Akhi, A. H., Talukder, M. Z. A. and Mujahidi, T. A.
(2018). Combining ability and heterosis study in maize (Zea mays L.) hybrids at different environments. Bangladesh J. Agril. Res. 43(1): 125-134.

Pansey, V.G. and Sukhante, P.V. (1985). "Statistical Methods for Agricultural Workers." I.C.A.R., New Delhi, pp.250-297.

Ratna B. D., Rajitha, A., Lal, A. M. and Srinivasa, V.R. (2014). Heterosis and combining ability for grain yield and yield component traits in maize ( $\mathrm{Zea}$ mays L.). Ele. J. of Plant Breeding., 5(3): 378-384

Sprague, G.F. and Tatum, L. (1942). General and specific combining ability in single crosses of Corn. J. American Sci. Agron., 34: 923-932.

Yerva, S. R., Sekhar, T.C., Allam, C.R. and Krishnan, V. (2016). Combining ability studies in maize (Zea mays L.) for yield and its attributing traits using Griffing's diallel approach. Ele. J. of Plant Breeding., 7(4): 1046-1055.

\section{How to cite this article:}

Bharat. G. B., P. B. Wadikar, M. V. Dhuppe and Thakur. N. R. 2020. Combining Ability Analysis for Yield and Yield Contributing Traits in Maize (Zea mays L.) Hybrids. Int.J.Curr.Microbiol.App.Sci. 9(06): 1234-1241. doi: https://doi.org/10.20546/ijcmas.2020.906.152 